\title{
Repercussões otorrinolaringológicas do abuso de cocaína e/ou crack em dependentes de drogas
}

\author{
A.C.N. Nassif Filho, S.G. Bettega, S. Lunedo, J.E. Maestri, F. Gortz \\ Trabal ho realizado na Santa Casa de Misericórdia de Curitiba, PR.
}

\begin{abstract}
RE SUMO - A prevalência de pacientes viciados em cocaína e/ou crack parece estar aumentando; portanto, o médico deve estar preparado para diagnosti car o uso de drogas.

Ов етіvos. Determinar se exi stem sinaistípi cos de uso de cocaína e/ou crack no trato respi ratório superior ou na orofaringe em exame otorrinolaringológico comum. Orientar o médico no diagnóstico dos pacientes viciados nessas drogas.

Mé Todos. Foram estudados 18 pacientes viciados em cocaína e/ou crack, que estavam internados em clínica psiquiátrica, através de anamnese exame otorrinolaringológi co simples (rinoscopia anterior e orofaringoscopia).
\end{abstract}

\section{INTRODUÇÃO}

A cocaína é uma substância natural extraída da coca. Em épocas passadas, foi largamente usada como anestésico tópico em ci rurgias oftal mológicas e otorrinolaringológicas, possuindo propriedades vasoconstritoras. Chega ao consumidor nas formas de um sal - cloridrato de cocaína - que pode ser aspirado ou, dissolvido em água, ser usado por via endovenosa. Há ainda a pasta de coca, que é um produto grosseiro, obtido nas primeiras fases de preparação; contém muitas impurezas e éfumada método pouco utilizado pel os dependentes da droga. As formas de uso da cocaína são, portanto: 1) aspirada (cujos efeitos começam em torno de três minutos); 2) injetada (seus efeitos iniciam-se em aproximadamente um mi nuto e meio); e 3) fumada (os efeitos demoram apenas al guns segundos para acontecer). A sua ação mais óbvia ocorre no sistema nervoso central, através de um bloqueio da recaptação da dopamina na fenda sináptica: sensações intensas de prazer, euforia e poder, diminuição da necessi dade de sono, aumento das sensações sexuais, redução do apetite, estado de hiperatividade com aceleração do pulso, aumento do ritmo respiratório, febre, hipertensão arterial, tremor nas mãos e agitação psicomotora.

O crack é um subproduto da cocaína. É obtido a partir da pasta de coca acrescida do bicarbonato de
Resultados. Foi impossível estabel ecer uma correlação entre o tempo de uso, quanti dade efreqüência no uso das drogas quanto aos sintomas e exame otorrinolaringológico.

Conclusão. Devido à inespecíficidade no exame otorri nolaringológi co do paci ente vi ciado em cocaína e/ou crack, é necessário que o médico suspei te do abuso de drogas na história clínica; caso contrário, poderá não realizar este importante diagnóstico.

UNITERMOS: Toxicologia. Cocaína. Otorrinolaringologia

sódi o, sendo comercializado na forma de pequenas pedras porosas. Fumado em pequenos cachimbos de fabri cação caseira ou através da inal ação do seu vapor, produz um efeito de frenética euforia e intensa excitação, sendo que uma pedra não rende mais do que duas horas de euforia. Seu início de ação se faz em aproximadamente oito segundos. Quando a pedra se esgota, sobrevem a exaustão e o usuário entra em sono profundo. Sendo uma droga de uso muito simples e muito barata, facilita o acesso à crianças de camadas pobres da população. O crack é mais potente e prejudicial do que a cocaína inalada ou injetada.

$\mathrm{Na}$ prática médica tem-se observado um aumento crescente na inci dência de pacientes dependentes de drogas, a despei to das constantes campanhas publicitárias que visam coi bir o seu uso. A cocaína e o crack são drogas que lesionam uma grande área do trato aerodigestivo superior, abrangendo a mucosa nasal, o septo nasal, os cornetos, a faringe, a mucosa oral, a laringe e, até mesmo, a região superior do esôfago. Isso ocorre devido não apenas aos seus efeitos irritativo e vasoconstritor, mas também porque a inalação de gases quentes em uma mucosa anestesiada pode levar a uma queimadura. Além disso, diversas substâncias que fazem parte dos frascos usados para aquecer o crack - como tinta, material plástico, restos no recipi ente e outros - são inaladas, podendo ocasionar lesões às mucosas oronasais. 
O diagnóstico das lesões causadas pela cocaína e pelo crack não é fácil. As dificuldades começam a partir da história, que comumente é confusa ou incoerente, pois o paciente, na grande maioria dos casos, omi te o uso da substância. A sintomatol ogia é inespecífica. $\mathrm{Na}$ literatura, encontram-se vários relatos em que os pacientes apresentam um quadro clínico que mimetiza uma série de outras patologias, como a granulomatose de Wegener, infecções por fungos ebactérias, I infoma nasossinusal, traumatismos, desordens neurológicas, etc.

\section{PACIENTES E MÉTODOS}

O estudo foi realizado em pacientes internados no mês de março de 1999 no Hospital Pinel, em Curitiba, que além do atendimento às diversas patol ogi as psiquiátricas, presta cui dados médi cos à pacientes dependentes de drogas e álcool. Os pacientes foram sel ecionados dentre os internados de maneira inteiramente aleatória. Todos estavam internados sob regime integral, com o fim específico de deixarem o abuso de cocaína e/ou crack. Todos os pacientes foram submetidos a um questionário e a um exame clínico.

Eles eram questionados quanto ao tempo de uso da cocaí na e/ou crack, quanto à quantidade de droga que o paciente costumava utilizar, quanto à freqüência no seu uso e há quanto tempo el e a utilizara pela última vez. Os pacientes dependentes de cocaína eram, ainda, questi onados quanto à forma noseu uso (fumada, aspirada ou injetada endovenosamente); se fosse na forma aspirada, o paciente era questionado se el e preferia usar al gum lado do nariz para fazê-lo. Por fim, os pacientes eram estimulados a responder positiva ou negativamente se apresentavam os seguintes sintomas, na época em que utilizavam a droga: voz anasalada, dor de garganta, obstrução nasal , rinor réi a, sangramento nasal, disfonia, disfagia, tosse, globus faríngeo, perda de olfato e perda do paladar.

O exame consistiu em or ofaringoscopia e rinoscopia anterior com espéculo nasal, no qual se utilizou um fotóforo. Na rinoscopia anterior, oexaminador determinava: 1) a coloração da mucosa (normal, empalidecida ou hiperemiada); 2) edema (presente ou ausente); 3 ) secreção (ausente, serosa, mucopurulenta ou crostosa); 4) desvio de septo nasal (à direita, à esquerda ou sem desvio); e 5) o aspecto do corneto inferior (normal, hipertrófico ou atrófico).

\section{RE SULTADOS}

Os autores pesquisaram as repercussões otorrinolaringológicas da cocaína e/ou crack em 18

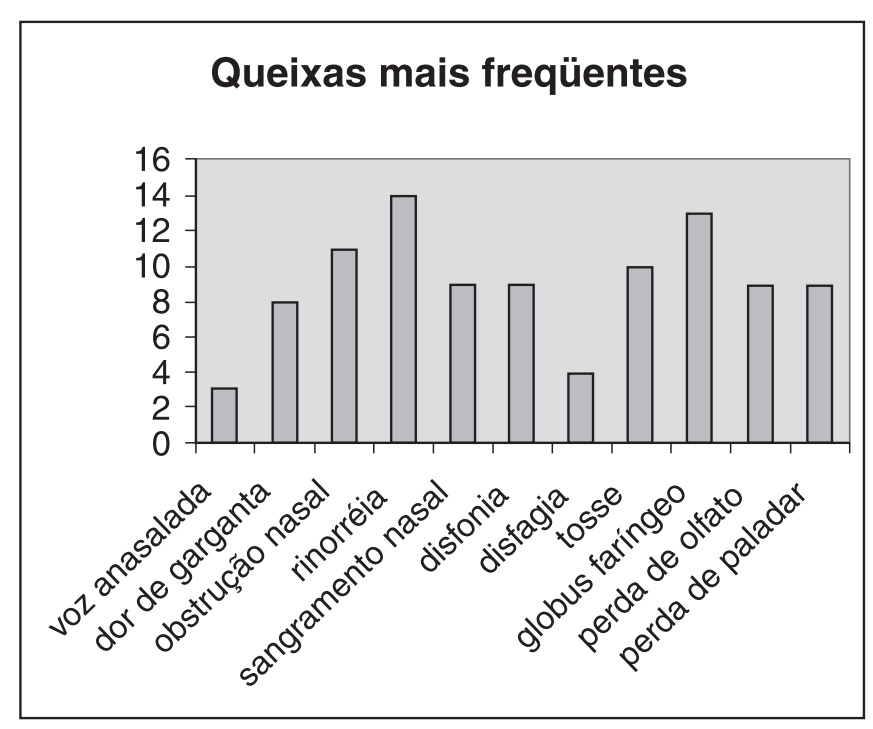

Fig. 1 - Fr reqüência das quei xas em 18 paci entes vi ciados em cocaína e/ ou crack.

pacientes, cuja idade variou entre 17 e 37 anos, sendo, em média, de 25,1 anos. Doze pacientes eram viciados em cocaína e crack; quatro, em cocaína; e dois pacientes faziam o abuso do crack isoladamente. Portanto, 16 pacientes eram viciados em cocaína e 14 em crack. Percebeu-se que os pacientes que afirmavam ter feito o abuso deambas as substânci as não as utilizavam em associação. A grande maioria substituíra a cocaína pelo crack, devido ao seu menor custo ou em decorrência dos efeitos mais pronunciados do crack no usuário.

Os pacientes viciados em cocaína afirmaram fazer o uso diário de cerca de 3,8 gramas, em média, com variação de 1 a 10 gramas por dia. Todos a utilizavam na forma aspirada e nenhum o fazia endovenosamente ou fumando-a. E m rel ação à freqüência no seu uso, 13 pacientes $(81,3 \%)$ afirmavam utilizar a cocaína todos os dias, e o restante, três pacientes (18,7\%), a utilizavam numa freqüência igual ou mai or que quatro vezes por semana. A freqüência no uso do crack era semelhante: dos 14 pacientes vi ciados nesta droga, $11(78,6 \%)$ a utilizavam todos os dias e os três pacientes restantes $(21,4 \%)$ afirmaram utilizá-la numa freqüência de cerca de cinco vezes por semana. A quantidade variou de 1 a 15 gramas, sendo, em média, de 5,2 gramas de crack por dia.

Dos 18 paci entes viciados em cocaína e/ou crack, responderam afirmativamente para os sintomas: (Fig. 1)

- voz anasalada: três pacientes (16,7\%);

- dor de garganta: oito pacientes $(44,4 \%)$;

- obstrução nasal: 11 pacientes $(61,1 \%)$;

- rinorréia: 14 pacientes (77,8\%);

- sangramento nasal: nove pacientes (50\%); 


\begin{tabular}{|c|c|c|c|c|}
\hline & \multicolumn{2}{|c|}{ Obstrução nasal } & \multicolumn{2}{|c|}{ Rinorréia } \\
\hline & Sim & Não & Sim & Não \\
\hline A & 3 & 2 & 4 & 1 \\
\hline B & 5 & 2 & 6 & 1 \\
\hline C & 2 & 2 & 4 & - \\
\hline \multicolumn{5}{|c|}{$\begin{array}{l}\text { A) Até } 2 \text { anos ( } 5 \text { pacientes) } \\
\text { B) De } 2 \text { a } 6 \text { anos ( } 7 \text { pacientes) } \\
\text { C) Mais de } 6 \text { anos ( } 4 \text { pacientes) } \\
\text { Total: } 16 \text { pacientes }\end{array}$} \\
\hline
\end{tabular}

Tabela 2 - Quanto à quantidade de cocaína que o paciente costumava utilizar:

\begin{tabular}{|ccccc|}
\hline & \multicolumn{2}{c}{ Obstrução nasal } & \multicolumn{2}{c|}{ Rinorréia } \\
A & Sim & Não & Sim & Não \\
B & 3 & 4 & 6 & 1 \\
C & 2 & 2 & 4 & - \\
\hline
\end{tabular}

A) Até 2 gramas/dia (7 pacientes)

B) De 2,1 a 4 gramas/dia (4 pacientes)

C) Mais que 4 gramas/dia (5 pacientes) Total: 16 pacientes

- disfonia: nove pacientes (50\%);

- disfagia: quatro pacientes (22,2\%);

- tosse: 10 pacientes (55,5\%);

- globus faríngeo: 13 pacientes (72,2\%);

- perda de ol fato: nove pacientes (50\%);

- perda do paladar: nove pacientes (50\%).

À or ofaringoscopia, houve o achado de hipertrofia amigdaliana de graus variados em quatro pacientes (22,2\%). Nos demais 14 pacientes $(77,8 \%)$, a orofaringe revelou-se sem quaisquer alterações.

À rinoscopia, os seguintes achados foram encontrados (sendo aqui contabilizados somente os resultados dos pacientes viciados em cocaína; 16 pacientes, no total):

Col oração da mucosa:

* NORMAL em três pacientes (18,75\%)

* HI PE RE M I ADA em sete pacientes (43,75\%)

* EMPALIDECIDA em seis pacientes (37,5\%)

E dema:

* PRESE NTE em 10 pacientes (62,5\%)

* AUSE NTE em seis pacientes (37,5\%)

Secreção:

* MU COPURULE NTA em dois pacientes (12,5\%)

* CROSTOSA em cinco pacientes (31,25\%)

* SEROSA em dois pacientes (12,5\%)

* AUSENTE em sete pacientes (43,75\%) Septo nasal:

* DESVIO À DIREITA em seis pacientes (37,5\%)

* DESVIOÀ ESQUERDA emquatropacientes (25\%)

* NORMAL em seis pacientes (37,5\%)
Aspecto do corneto inferior:

* HIPERTRÓFICO BILATERAL em oito pacientes (50\%)

* HIPERTRÓFICO UNILATERAL em três pacientes $(18,75 \%)$

* ATRÓFICO em nenhum paciente

* NORMAL em cinco pacientes (31,25\%)

N enhum paciente foi considerado como "normal" para todos os critérios adotados. Em um paciente foi descrito um achado de neovascularização do terço médio do septo nasal.

Os pacientes também foram questionados se preferiam algum lado do nariz para aspi rar a cocaína ou se utilizavam ambos os lados. Esse dado foi correlacionado com a descrição do septo nasal. Os resultados foram os seguintes: 12 pacientes (75\%) utilizavam o lado oposto ao desvio septal ou ambos os lados quando este era normal, três pacientes $(18,75 \%)$ afi rmaram aspi rar a cocaína por ambos os lados, mas apresentaram desvio septal à rinoscopia, dois paci entes (12,5\%) preferiam utilizar um dos lados do nariz, mesmo apresentando um septo normal ao exame e um paci ente $(6,25 \%)$ utilizava apenas um lado do nariz para aspirar a cocaína; paradoxalmente, o septo nasal foi descrito como desviado para esse mesmo lado.

\section{DISCUSSÃO}

Observou-se uma sintomatol ogia bastante exuberante, entretanto, inespecífica entre os viciados em cocaína e/ou crack. Além disso, a rinoscopia anterior demonstrou que, na maioria dos casos, há uma anormalidade da cavidade nasal; todavia, o aspecto da mucosa é, igualmente, inespecífico.

Os dezesseis pacientes viciados em cocaína foram divididos em subgrupos e correl acionados aos dois sintomas nasais mais freqüentes: obstrução nasal e rinorréia. (Tabelas 1 e 2)

N ota-se que não é possível correl acionar otempo de uso e nem a quantidade de cocaína utilizada com a sintomatol ogia. I sso foi verificado não apenas em rel ação à obstrução nasal e à rinorréia, como também em relação aos outros sintomas arrolados no questionário.

Posteri ormente, os pacientes viciados em cocaína foram subdi vididos e comparados aos achados da rinoscopia:

Não se encontrou especificidade entre a exposição à droga e a rinoscopia anterior, como se pode observar nas Tabelas 3, 4 e 5.

A propor ção de pacientes com desvio de septo nos pacientes viciados em cocaína está elevada em rel ação à população em geral. Dos 16 pacientes, 10 $(62,5 \%)$ apresentaram desvio de septo. Os autores 


\begin{tabular}{|c|c|c|c|c|c|c|c|c|c|c|c|c|}
\hline & \multicolumn{3}{|c|}{ Mucosa } & \multicolumn{4}{|c|}{ Secreção } & \multicolumn{2}{|c|}{ Edema } & \multicolumn{3}{|c|}{ Corneto inf. } \\
\hline & 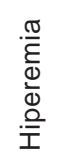 & $\frac{N}{\frac{N}{O}}$ & $\begin{array}{l}\overline{\widetilde{\sigma}} \\
\text { Eे } \\
\text { Z }\end{array}$ & 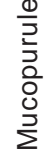 & 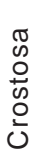 & 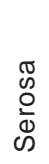 & 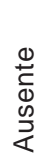 & 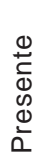 & $\begin{array}{l}\stackrel{0}{*} \\
\stackrel{0}{0} \\
\stackrel{0}{D} \\
\stackrel{0}{<}\end{array}$ & 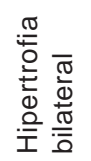 & 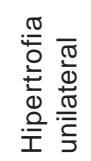 & $\begin{array}{l}\bar{\pi} \\
\stackrel{\Sigma}{\vdots} \\
\text { 으 }\end{array}$ \\
\hline A & 2 & 2 & 1 & - & - & - & 5 & 3 & 2 & 5 & - & - \\
\hline B & 3 & 2 & 2 & 1 & 3 & 2 & 1 & 4 & 3 & 1 & 2 & 4 \\
\hline C & 2 & 2 & - & 1 & 2 & - & 1 & 3 & 1 & 2 & 1 & 1 \\
\hline \multicolumn{13}{|c|}{$\begin{array}{l}\text { A) Até } 2 \text { anos ( } 5 \text { pacientes) } \\
\text { B) De } 2 \text { a } 6 \text { anos ( } 7 \text { pacientes) } \\
\text { C) Mais de } 6 \text { anos ( } 4 \text { pacientes) } \\
\text { Total: } 16 \text { pacientes }\end{array}$} \\
\hline
\end{tabular}

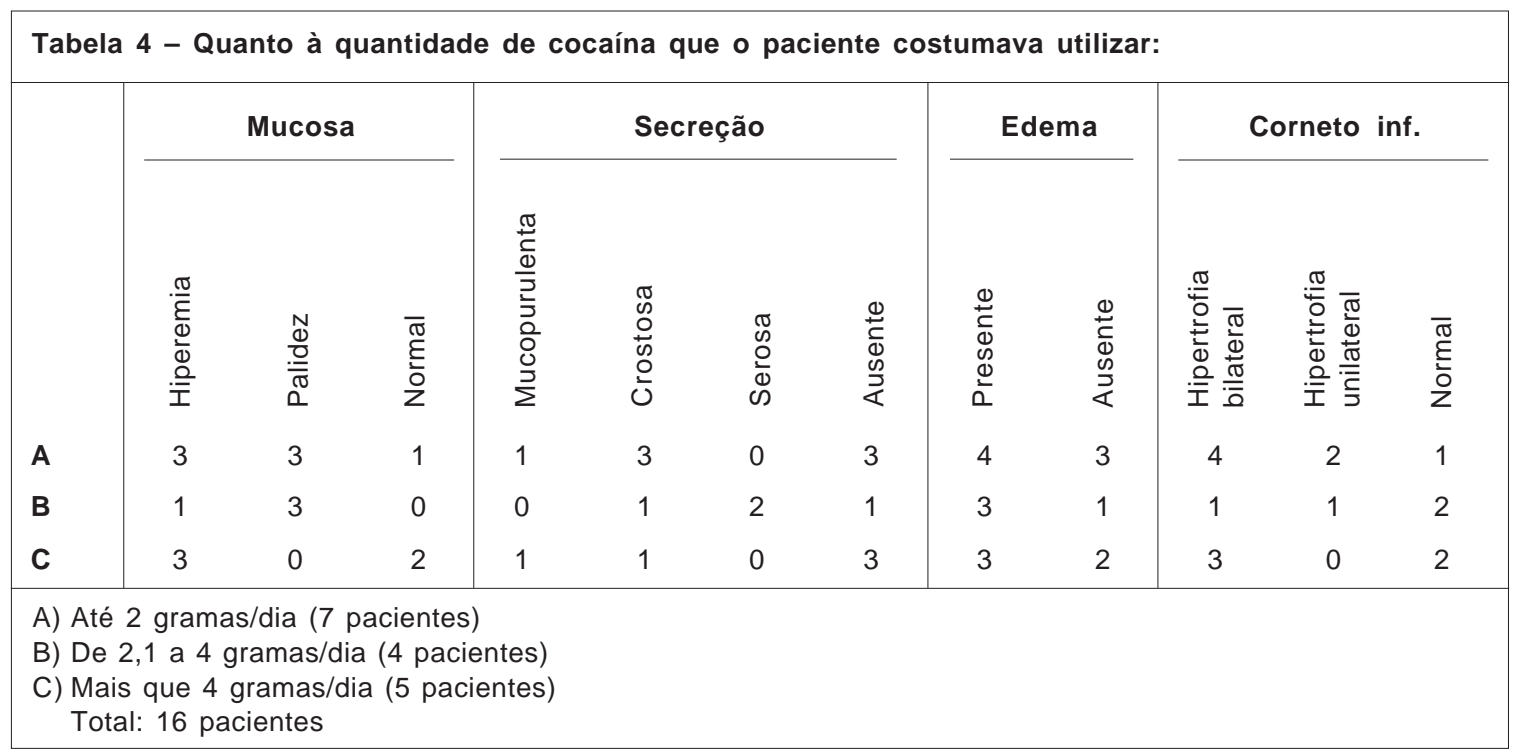

\begin{tabular}{|c|c|c|c|c|c|c|c|c|c|c|c|c|}
\hline & & uco & & & & & & & & & rneto & \\
\hline & 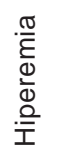 & $\frac{N}{\frac{N}{\overparen{D}}}$ & $\begin{array}{l}\overline{\widetilde{\pi}} \\
\text { ¿े } \\
\text { Z }\end{array}$ & $\begin{array}{l}\frac{0}{3} \\
\frac{1}{3} \\
\frac{0}{0} \\
\frac{0}{0} \\
\frac{0}{2}\end{array}$ & 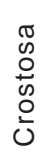 & 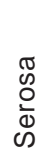 & $\begin{array}{l}\stackrel{0}{ \pm} \\
\stackrel{2}{D} \\
\stackrel{\infty}{2} \\
\frac{1}{<}\end{array}$ & $\begin{array}{l}\stackrel{1}{ \pm} \\
\stackrel{0}{0} \\
0 \\
\stackrel{\infty}{L}\end{array}$ & 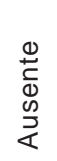 & 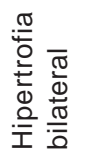 & 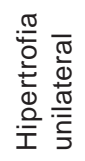 & $\begin{array}{l}\overline{\tilde{\sigma}} \\
\frac{\bar{c}}{2}\end{array}$ \\
\hline A & 3 & 2 & 2 & 2 & 1 & 1 & 3 & 4 & 3 & 4 & 1 & 2 \\
\hline B & 1 & 2 & 1 & - & 2 & - & 2 & 4 & - & 1 & 1 & 2 \\
\hline C & 3 & 2 & - & - & 2 & 1 & 2 & 2 & 3 & 3 & 1 & 1 \\
\hline \multicolumn{13}{|c|}{$\begin{array}{l}\text { A) Há } 4 \text { meses ou mais ( } 7 \text { pacientes) } \\
\text { B) De } 1 \text { há } 4 \text { meses ( } 4 \text { pacientes) } \\
\text { C) Até há } 1 \text { mês ( } 5 \text { pacientes) } \\
\text { Total: } 16 \text { pacientes }\end{array}$} \\
\hline
\end{tabular}




\begin{tabular}{|c|c|c|c|c|c|c|}
\hline & \multicolumn{2}{|c|}{ Disfonia } & \multicolumn{2}{|c|}{ Tosse } & \multicolumn{2}{|c|}{ Globus faríngeo } \\
\hline & Sim & Não & Sim & Não & Sim & Não \\
\hline A & 2 & 5 & 34 & 5 & 2 & \\
\hline B & 5 & 2 & 52 & 6 & 1 & \\
\hline \multicolumn{7}{|c|}{$\begin{array}{l}\text { A) Até um ano ( } 7 \text { pacientes) } \\
\text { B) Por mais de um ano ( } 7 \text { pacientes) } \\
\text { Total: } 14 \text { pacientes }\end{array}$} \\
\hline
\end{tabular}

não conseguiram encontrar uma rel ação de causa e efeito para este dado. Por outro lado, os pacientes, em geral, afirmavam utilizar o lado do nariz sem desvio, ou ambos os I ados caso não houvesse desvio, o que pareceu facil mente expli cável para os exami nadores: o paciente utiliza a cavidadenasal onde há maior amplitudee, provavel mente, maior absorção da droga.

Os quatorze pacientes viciados em crack foram subdivididos e comparados aos seguintes sintomas: disfonia, tosse e globus faríngeo.

A pesar de haver, aparentemente, uma proporção significativa de pacientes com tosse e que utilizavam uma quantidade de crack superior a três gramas por dia, não é possível estabel ecer uma rel ação específica entre a exposição a esta droga e os sintomas apresentados pel o paciente.

\section{CONCLUSÃO}

Os autores concluem que, em pacientes viciados em cocaína e/ou crack, é necessário que o médico tenha um al to índice de suspei ta clínica em relação ao abuso dessas substâncias esti mul antes do sistema nervoso central. Os pacientes podem apresentar uma sintomatol ogi a bastante exuberante, mas que é inespecífica. Além disso, o seu exame otorrinolaringológico pode ser praticamente normal ou com alterações bastante pronunciadas, mas igualmente inespecíficas.

Devido à ausência de uma apresentação clínica típica, o médico poderá, por exemplo, realizar o diagnóstico de rinite alérgica ou de rinite mucocatarral. Deve-se, portanto, estar atento no atendimento a esses pacientes, pois freqüentemente omitem o fato de fazer o abuso de tais substâncias. A anamnese deve ser minuciosa e a relação médicopaciente, bem desenvolvida; caso contrário, não é possível, na maioria das vezes, realizar o diagnóstico de abuso de drogas.

\section{SUMMARY}

\section{Otorhinolaryngological repercussions of the abuse of cocaine and/or crack in patients ad- dicted to drugs}

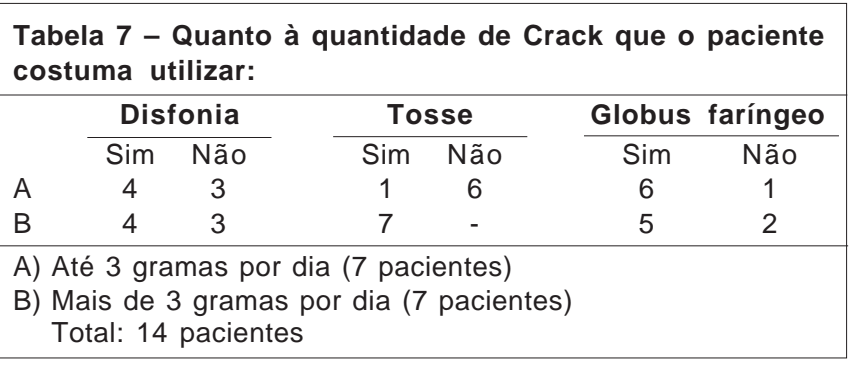

Purpose. To assess if there are typical otorhinolaryngological findings in patientes addicted to cacaineand/ or crack.

METHODS. E ighteen patients who wereaddicted to cacaine and/ or crack, were studied. Their clinical history was obtained and were submitted to an otorhinol aryngol ogical examination.

RESULTS. It was impossible to find out a correlation between the time the patient is addicted to the drug, the amount of drug the patient is used to inhale, the frequency or the last time he used it and the nose and throat symptoms, as well as those factors and theotor hi nol aryngol ogi cal examination.

CONCLUSION. Owing totheunspecificity on clinical findings during the otorhinolaryngological examinati on, it is necessary that thephysi ci an suspects the abuse of drugs when he is performing the clinical history; otherwise, he will not reach this important diagnosis. [Rev Ass Med Brasil 1999; 45(3): 237-41.]

KEY WORDS: Toxicology. Cocaine. Otorhinolaringol ogy.

\section{REFERÊ NCIAS BIBLIOGRÁFICAS}

1. Yanagisawa E \& Latorre R. Endoscopic view of cocaine rhinitis. ENT-Ear, Nose \& Throat J ournal. Mar 1996. pp. 128-9.

2. Meleca RJ, Burgio DL, Carr RM \& Lolachi CM. Mucosal injuries of the upper aerodigestive tract after smoking crack or freebase cocaine. Laryngoscope 1997; 107:620-25.

3. Armstrong $M$, Shikani AH. Nasal septal necrosis mimicking Wegener's granulomatosis in a cocaine abuser. ENT-Ear, Nose \& Throat J ournal 1996; 75(9):623-26.

4. Kridel RWH, Foda H, Lunde KC. Septal perforation repair with acellular human dermal allograft. Arch Otolaryngol Head Neck Surg 1998; 124:73-78.

5. Becker GD \& Hill S. Midline granuloma due to illicit cocaine use. Arch Otolaryngol Head Neck Surg 1988; 114: 90-91.

6. Kuriloff DB \& Kimmelman CP. Osteocartilaginous necrosis of the sinonasal tract following cocaine abuse. Laryngoscope 1989; 99: 918-24.

7. Bezmalinovic Z, Gonzalez M \& Farr C. Oropharyngeal injury possibly due to free-base cocaine. N Engl J Med 1988; 319: 1420-21.

8. Schweitzer VG. Osteolytic sinusitis and pneumomediastinum: Deceptive otolaryngologic complications of cocaine abuse. Laryngoscope 1986; 96:206-10.

9. Sawicka EH \& Trosser A. Cerebrospinal fluid rhinorrhea after cocaine sniffing. Br Med J 1983; 286: 1476-77.

10. Vilensky W. Illicit and licit drugs causing perforation of the nasal septum. J For Sci 1982; 27(4):958-962. 